\title{
Intramuscular Ketamine to Treat Major Depressive Disorder: A Case Series of Forty Patients
}

\author{
Carl J Bonnett ${ }^{1, *}$, Rakesh Jain ${ }^{2}$, Cassie N Ross ${ }^{3}$, Desmond A Wallington ${ }^{1}$, and Tony R Schock ${ }^{1}$ \\ ${ }^{1}$ Klarisana Psychedelic Therapies, Denver, Colorado, USA \\ ${ }^{2}$ Clinical Professor, Department of Psychiatry, Texas Tech University School of Medicine- Permian Basin, Midland, Texas, USA \\ ${ }^{3}$ University of Michigan Medicine, Department of Pediatrics, Division of Pediatric Psychology, Ann Arbor, Michigan, USA
}

*Corresponding author: Carl J Bonnett, Klarisana Psychedelic Therapies, Denver, Colorado, USA, E-mail: cbonnett@klarisana.com

Received: 01 Sep, 2021 | Accepted: 06 Oct, 2021 | Published: 13 Oct, 2021

Citation: Bonnett CJ, Jain R, Ross CN, Wallington DA, Schock TR (2021) Intramuscular Ketamine to Treat Major Depressive Disorder: A Case Series of Forty Patients. J Psychiatry Ment Health 6(2): dx.doi.org/10.16966/2474-7769.145

Copyright: (C) 2021 Bonnett CJ, et al. This is an open-access article distributed under the terms of the Creative Commons Attribution License, which permits unrestricted use, distribution, and reproduction in any medium, provided the original author and source are credited.

\begin{abstract}
Introduction: While the use of ketamine to treat severe depression and post-traumatic stress disorder has been well documented in the literature, fewer studies have investigated the efficacy of Intramuscular (IM) ketamine to treat these conditions.

Methods: This case series included 40 patients with major depressive disorder who received this center's standard treatment of six IM ketamine treatments. Patients completed the Patient Health Questionnaire (PHQ-9), PTSD Checklist for DSM-V (PCL-5), and the Generalized Anxiety Disorder-7 (GAD-7) before ketamine administration and prior to the sixth session.

Results: Forty subjects (19 male, 21 female) participated in the study with a mean age of 36.9 years (range 18-67 years). On the measure of depression (PHQ-9), the mean score showed a 55\% reduction, with a Cohen's $d$ effect size of 1.71 ( $F=84.4, P<0.001)$. Measurement of PTSD (PCL-5) symptoms showed a 51\% decrease in mean score, with Cohen's $d$ effect size of 1.61 ( $F=73.4, P<0.001)$. The Generalized Anxiety Disorder (GAD-7) instrument showed a 51\% mean score decrease, with Cohen's $d$ size effect of $1.63(\mathrm{~F}=66.1, \mathrm{P}<0.001)$.

Conclusions: The results of the series of patients presented in this series show that the immediate results of intramuscular therapy are comparable to those found using intravenous therapy.
\end{abstract}

Keywords: Patient Health Questionnaire (PHQ-9); Depression; Intramuscular ketamine

\section{Introduction}

The use of ketamine to treat severe depression and Post-Traumatic Stress Disorder (PTSD) has been well documented in the medical literature [1-8]. Many studies have looked at the use of Intravenous (IV) ketamine, but fewer have specifically looked at the use of Intramuscular (IM) ketamine to treat mental health conditions [9-11]. In order to increase access to ketamine therapy during the COVID-19 pandemic that unfolded in 2020, the centers described in this report joined the Colorado Health First network (CHF). CHF is Colorado's Medicaid program. Reimbursement rates from $\mathrm{CHF}$ are much lower than what was previously received with an IV cash-pay model, so it was imperative to adopt a strategy to make operations more cost effective while at the same time maintaining clinical effectiveness. In order to accomplish this, our centers converted from a treatment model based on IV ketamine to one based on IM ketamine. These operational changes resulted in a decrease in costs and an increase in the ability to provide access to psychedelic treatments to a much larger group of patients. In this case series we present our treatment results on a series of forty sequential patients who had a diagnosis of major depressive disorder and who received our new IM protocol. All patients described received our standard clinical treatment protocol as outlined in the procedures. No experimental component was added outside of the standard clinical protocol. We will discuss how these results compare favorably to both our previous published results and the results of other investigators.

\section{Methods}

\section{Study participants}

This case series describes forty sequential patients who were treated at the participating centers. All patients in this series had a diagnosis of major depressive disorder and had health first Colorado as their payor source. All of the patients received our standard treatment protocol and there was no experimental component added to their treatment plan. The mean age of the subjects was 36.9 years (range 1867 years). There were 19 male subjects and 21 female subjects. None of the patients carried any diagnosis of psychosis.

\section{Procedures}

Each patient had an intake screening by one of our licensed mental health professionals and was determined to be an appropriate candidate for ketamine therapy. All the patients in this series already 
Table 1: Individual data pre and post ketamine scores.

\begin{tabular}{|c|c|c|c|c|c|c|c|c|c|c|c|}
\hline \multicolumn{12}{|c|}{ Individual Patient Data } \\
\hline & \multirow{2}{*}{ Gender } & \multirow{2}{*}{ Weight (kg) } & \multirow{2}{*}{ Age } & \multirow{2}{*}{$\begin{array}{c}\text { Final Dose } \\
\text { (mg) }\end{array}$} & \multirow{2}{*}{$\begin{array}{c}\text { Final Dose } \\
\text { (mg/kg) }\end{array}$} & \multicolumn{2}{|c|}{ PHQ-9 Score } & \multicolumn{2}{|c|}{ PCL-5 Score } & \multicolumn{2}{|c|}{ GAD-7 Score } \\
\hline & & & & & & $\begin{array}{c}\text { Pre- } \\
\text { Ketamine }\end{array}$ & $\begin{array}{c}\text { Post- } \\
\text { Ketamine }\end{array}$ & $\begin{array}{c}\text { Pre- } \\
\text { Ketamine }\end{array}$ & $\begin{array}{c}\text { Post- } \\
\text { Ketamine }\end{array}$ & $\begin{array}{c}\text { Pre- } \\
\text { Ketamine }\end{array}$ & $\begin{array}{c}\text { Post- } \\
\text { Ketamine }\end{array}$ \\
\hline Patient 1 & $\mathrm{~F}$ & 50 & 49 & 70 & 1.4 & 13 & 8 & 50 & 35 & 13 & 6 \\
\hline Patient 2 & $\mathrm{~F}$ & 48 & 52 & 160 & 3.3 & 17 & 4 & 54 & 13 & 17 & 3 \\
\hline Patient 3 & $\mathrm{~F}$ & 99 & 55 & 180 & 1.8 & 21 & 15 & 48 & 38 & 17 & 17 \\
\hline Patient 4 & $M$ & 82 & 26 & 180 & 2.2 & 23 & 0 & 68 & 31 & 21 & 10 \\
\hline Patient 5 & $M$ & 73 & 53 & 160 & 2.2 & 16 & 13 & 29 & 44 & 12 & 11 \\
\hline Patient 6 & $\mathrm{~F}$ & 93 & 27 & 140 & 1.5 & 18 & 13 & 57 & 30 & 13 & 4 \\
\hline Patient 7 & $\mathrm{~F}$ & 65 & 29 & 180 & 2.8 & 15 & 7 & 41 & 9 & 18 & 7 \\
\hline Patient 8 & $\mathrm{M}$ & 111 & 31 & 160 & 1.4 & 14 & 8 & 15 & 16 & 16 & 5 \\
\hline Patient 9 & $\mathrm{~F}$ & 79 & 30 & 180 & 2.3 & 23 & 6 & 73 & 24 & 21 & 7 \\
\hline Patient 10 & $M$ & 78 & 59 & 160 & 2.1 & 23 & 4 & 40 & 12 & 17 & 3 \\
\hline Patient 11 & $M$ & 86 & 44 & 160 & 1.9 & 25 & 9 & 40 & 26 & 9 & 7 \\
\hline Patient 12 & $\mathrm{~F}$ & 51 & 18 & 120 & 2.4 & 21 & 4 & 56 & 17 & 18 & 4 \\
\hline Patient 13 & $\mathrm{~F}$ & 100 & 49 & 180 & 1.8 & 17 & 10 & 34 & 19 & 9 & 4 \\
\hline Patient 14 & $M$ & 146 & 45 & 180 & 1.2 & 8 & 9 & 41 & 19 & 18 & 7 \\
\hline Patient 15 & $\mathrm{M}$ & 102 & 31 & 180 & 1.8 & 15 & 3 & 59 & 14 & 17 & 6 \\
\hline Patient 16 & $M$ & 99 & 36 & 180 & 1.8 & 11 & 9 & 34 & 32 & 10 & 7 \\
\hline Patient 17 & $M$ & 85 & 31 & 140 & 1.6 & 10 & 0 & 52 & 5 & 12 & 1 \\
\hline Patient 18 & $\mathrm{M}$ & 80 & 34 & 160 & 2 & 20 & 2 & 52 & 12 & 20 & 6 \\
\hline Patient 19 & $M$ & 79 & 52 & 100 & 1.3 & 17 & 13 & 27 & 15 & 7 & 16 \\
\hline Patient 20 & $\mathrm{~F}$ & 62 & 18 & 160 & 2.6 & 27 & 19 & 67 & 66 & 18 & 13 \\
\hline Patient 21 & $M$ & 61 & 30 & 140 & 2.3 & 12 & 2 & 50 & 12 & 10 & 5 \\
\hline Patient 22 & $M$ & 73 & 40 & 160 & 2.2 & 13 & 7 & 56 & 15 & 7 & 7 \\
\hline Patient 23 & $\mathrm{M}$ & 68 & 20 & 140 & 2.1 & 19 & 12 & 39 & 32 & 13 & 13 \\
\hline Patient 24 & $\mathrm{M}$ & 122 & 67 & 110 & 0.9 & 18 & 6 & 40 & 18 & 7 & 3 \\
\hline Patient 25 & $\mathrm{~F}$ & 77 & 42 & 160 & 2.1 & 1 & 1 & 4 & 5 & 6 & 2 \\
\hline Patient 26 & $\mathrm{~F}$ & 91 & 28 & 180 & 2 & 13 & 9 & 47 & 25 & 9 & 7 \\
\hline Patient 27 & $\mathrm{~F}$ & 62 & 34 & 140 & 2.3 & 17 & 1 & 35 & 6 & 12 & 3 \\
\hline Patient 28 & $\mathrm{M}$ & 76 & 20 & 160 & 2.1 & 19 & 5 & 15 & 8 & 11 & 6 \\
\hline Patient 29 & $\mathrm{M}$ & 85 & 53 & 140 & 1.6 & 20 & 13 & 47 & 41 & 18 & 14 \\
\hline Patient 30 & $\mathrm{~F}$ & 77 & 23 & 180 & 2.3 & 19 & 5 & 57 & 18 & 21 & 7 \\
\hline Patient 31 & $\mathrm{~F}$ & 52 & 22 & 120 & 2.3 & 10 & 5 & 50 & 23 & 20 & 7 \\
\hline Patient 32 & $\mathrm{~F}$ & 53 & 22 & 180 & 3.4 & 8 & 1 & 42 & 20 & 10 & 6 \\
\hline Patient 33 & $\mathrm{~F}$ & 71 & 25 & 160 & 2.3 & 18 & 9 & 68 & 44 & 21 & 11 \\
\hline Patient 34 & $\mathrm{~F}$ & 81 & 37 & 180 & 2.2 & 16 & 8 & 63 & 24 & 15 & 7 \\
\hline Patient 35 & $M$ & 69 & 36 & 180 & 2.6 & 3 & 8 & 17 & 26 & 6 & 8 \\
\hline Patient 36 & $\mathrm{~F}$ & 77 & 48 & 140 & 1.8 & 10 & 1 & 43 & 14 & 18 & 6 \\
\hline Patient 37 & $\mathrm{~F}$ & 90 & 39 & 100 & 1.1 & 20 & 7 & 50 & 8 & 17 & 4 \\
\hline Patient 38 & $\mathrm{~F}$ & 101 & 58 & 80 & 0.8 & 13 & 15 & 37 & 34 & 4 & 3 \\
\hline Patient 39 & $M$ & 93 & 31 & 140 & 1.5 & 17 & 4 & 53 & 11 & 20 & 6 \\
\hline Patient 40 & $\mathrm{~F}$ & 101 & 30 & 120 & 1.2 & 17 & 8 & 40 & 17 & 17 & 5 \\
\hline
\end{tabular}

had an established diagnosis of major depressive disorder. Each patient had three psychological scoring instruments administered before, and at the end of an induction series of six ketamine treatment sessions. Patients were administered the patient health questionnaire (PHQ-9), PTSD checklist for DSM-V (PCL-5) and generalized anxiety disorder scale (GAD-7) prior to the first ketamine session. Just prior to each patient's sixth session the same three instruments were administered again [11-16].
The participants all received treatment according to our standard clinical protocol. Per our protocol, participants received an initial series of six IM ketamine sessions spread over three weeks. Patients started with an initial dose of $1 \mathrm{mg} / \mathrm{kg}$ with a maximum of $60 \mathrm{mg}$ on the first session. The total dose is divided into two equal doses separated by fifteen minutes. If a patient appears to be having an overly profound psychomimetic experience after the first injection, then the clinician may refrain from administering the second dose, though

Citation: Bonnett CJ, Jain R, Ross CN, Wallington DA, Schock TR (2021) Intramuscular Ketamine to Treat Major Depressive Disorder: 
Table 2: Pre and post ketamine infusion scores.

\begin{tabular}{|c|c|c|c|c|c|c|c|c|}
\hline \multicolumn{9}{|c|}{ Psychological Screening Instrument Results } \\
\hline & Pre-Ketamine & SD & Post-Ketamine & SD & $\mathbf{F}$ & $\mathbf{P}$ & Chi-Squared & Change \\
\hline PHQ-9 & 15.9 & 5.6 & 7.1 & 4.6 & 84.4 & $<0.001$ & 9.3 & $-55 \%$ \\
\hline PCL-5 & 44.8 & 15.3 & 22.0 & 12.9 & 73.4 & $<0.001$ & 9.2 & $-51 \%$ \\
\hline GAD-7 & 14.1 & 5.0 & 6.9 & 3.7 & 66.1 & $<0.001$ & 9.3 & $-51 \%$ \\
\hline
\end{tabular}

Table 3: Final ketamine dose calculation.

\begin{tabular}{|l|c|c|c|c|}
\hline \multicolumn{5}{|c|}{ Ketamine Doseage on Sixth Session } \\
\hline & Mean & SD & Median & Range \\
\hline $\begin{array}{l}\text { Dose } \\
\text { Calculation } \\
\text { (mg/kg) }\end{array}$ & 1.97 & 0.57 & 2.05 & $0.8-3.4$ \\
\hline $\begin{array}{l}\text { Final Dose } \\
\text { (mg) }\end{array}$ & 151.0 & 29.6 & 160.0 & $70-180$ \\
\hline
\end{tabular}

this is rare. After the first session, the dose of ketamine is increased incrementally to guide the patient to a point of enlightened experience that we refer to as the Psychotropic Therapeutic Response (PTR). This stepwise escalation is based on patient response and determined through a collaborative discussion between the providers and patients. Per protocol, the maximum dose that could be administered is $200 \mathrm{mg}$ total divided into two $100 \mathrm{mg}$ injections spaced fifteen minutes apart.

On the first visit, each patient has his/her weight measured and recorded. During each ketamine session, patients have vital signs checked prior to administration of ketamine, which include blood pressure, heart rate, pulse oximetry and respirations. They then have repeat vital signs documented before the second injection and one hour after the first injection. During each session, patients are monitored and observed by clinical personnel while receiving ketamine. A combination of direct monitoring and observation using high-quality video cameras is utilized. The video cameras allowed for better social distancing in the context of the COVID-19 pandemic [17-20].

\section{Results}

The individual demographic, dosing and pre/post-ketamine score results for each of the 40 patients are presented in table 1 . In this series, 39 of 40 patients $(98 \%)$ reported improvement in their depression symptoms. Pooled data of the mean scores of each survey instrument are shown on table 2. On the measure of depression (PHQ-9), the mean score showed a 55\% reduction from 15.9 to 7.1, with a Cohen's $d$ effect size of $1.71(\mathrm{~F}=84.4, \mathrm{P}<0.001)$. Measurement of PTSD (PCL-5) symptoms showed a decrease in mean score from 44.8 pre-treatment to a score of 22.0. This represents a $51 \%$ reduction, with Cohen's $d$ effect size of $1.61(\mathrm{~F}=73.4, \mathrm{P}<0.001)$. The generalized anxiety disorder (GAD-7) instrument showed a mean score of 14.1 pre-ketamine and decreased to a mean score of 6.9 after ketamine treatment. This represents a 51\% decrease, with Cohen's $d$ size effect of 1.63 ( $\mathrm{F}=66.1$, $\mathrm{P}<0.001)$.

Table 3 shows the mean dosage on the final ketamine session in terms of absolute dose and in terms of milligrams per kilogram of ketamine. Throughout the six sessions we progressively increase the doses to the desired experiential effect. The mean final doses of ketamine were $151 \mathrm{mg}$ in absolute dose and $1.97 \mathrm{mg}$ per kilogram of body weight.

\section{Discussion}

The results of the series of patients presented in this series show that the immediate results of intramuscular therapy are comparable to those found using intravenous therapy [1-3,7,20-24]. Interestingly, our results with this series are also comparable to a previous study we published that looked at the use of intravenous ketamine to treat PTSD in a series of combat veterans [20]. In that study we demonstrated a $97 \%$ positive response rate of the veterans to ketamine therapy. The primary diagnosis in that study was PTSD however whereas in this series, it is major depressive disorder. Nonetheless it is revealing that the results are similar given that each cohort was treated in the same facilities by the same clinical staff with only the route of delivery of ketamine changed.

Other authors have demonstrated response rates for patients with severe depression treated with ketamine ranging from approximately $60-80 \%$ [21-24]. The results of this case series suggest that delivery of ketamine through an IM route does not appear to be inferior to IV ketamine. Further studies that compare IM ketamine head-to-head with both IV and a new commercially available intranasal formulation would be very valuable [16]. Additionally, longitudinal studies to look at the long-term sustainability of the antidepressant effect of IM vs. IV ketamine would be valuable. Given that IM ketamine can be administered in a much more cost-effective manner, these results have significant public health ramifications with regards to increasing access to psychedelic therapies such as ketamine for the treatment of mood disorders.

\section{Acknowledgement}

Candy McKenna assisted with layout, data synthesis, and proofreading.

\section{References}

1. Murrough JW, losifescu DV, Chang LC, Al Jurdi RK, Green CE, et al. (2013) Antidepressant efficacy of ketamine in treatment-resistant major depression: a two-site randomized controlled trial. Am J Psychiatry 170: 1134-1142.

2. Murrough JW, Perez AM, Pillemer S, Stern J, Parides MK, et al. (2013) Rapid and longer-term antidepressant effects of repeated ketamine infusions in treatment-resistant major depression. Biol Psychiatry 74: 250-256.

3. Phillips JL, Norris S, Talbot J, Hatchard T, Ortiz A, et al. (2020) Single and repeated ketamine infusions for reduction of suicidal ideation in treatment-resistant depression. Neuropsychopharmacology 45: 606-612.

4. Price RB, losifescu DV, Murrough JW, Chang LC, Al Jurdi RK, et al. (2014) Effects of ketamine on explicit and implicit suicidal cognition: a randomized controlled trial in treatment-resistant depression. Depress Anxiety 31: 335-443. 
5. Grunebaum MF, Galfalvy HC, Choo TH, Keilp JG, Moitra VK, et al. (2018) Ketamine for rapid reduction of suicidal thoughts in major depression: amidazolam-controlled randomized clinical trial. Am J Psychiatry 175: 327-335.

6. Niciu MJ, Luckenbaugh DA, lonescu DF, Guevara S, Vieira RM, et al. (2014) Clinical predictors of ketamine response in treatmentresistant major depression. J Clin Psychiatry75: e417-e423.

7. Phillips JL, Norris $\mathrm{S}$, Talbot J, Birmingham $\mathrm{M}$, Hatchard $\mathrm{T}$, et al. (2019) Single, repeated, and maintenance ketamine infusions for treatment-resistant depression: a randomized controlled trial. Am J Psychiatry 176: 401-409.

8. Sanacora G, Frye MA, McDonald W, Mathew SJ, Turner MS, et al. (2017) A consensus statement on the use of ketamine in the treatment of mood disorders. JAMA Psychiatry 74: 399-405.

9. Dore J, Turnipseed B, Dwyer S, Turnipseed A, Andries J, et al. (2019) Ketamine Assisted Psychotherapy (KAP): patient demographics, clinical data and outcomes in three large practices administering ketamine with psychotherapy. J Psychoactive Drugs 51: 189-198.

10. Harihar C, Dasari P, Srinivas JS (2013) Intramuscular ketamine in acute depression: a report on two cases. Indian J Psychiatry 55: 186188

11. Kroenke K, Spitzer RL, Williams JBW (2001) The PHQ-9. J Gen Intern Med 16: 606-613.

12. Kroenke K, Spitzer RL (2002) The PHQ-9: a new depression diagnostic and severity measure. Psychiatric Annals 32: 509-515.

13. Blevins CA, Weathers FW, Davis MT, Witte TK, Domino JL (2015) The posttraumatic stress disorder checklist for DSM-5 (PCL-5): Development and initial psychometric evaluation. J Trauma Stress 28: $489-498$

14. Bovin MJ, Marx BP, Weathers FW, Gallaghar MW, Rodriguez P, et al. (2016) Psychometric properties of the PTSD checklist for diagnostic and statistical manual of mental disorders-fifth edition (PCL-5) in veterans. Psychol Assess 28: 1379-1391.

15. Spitzer RL, Kroenke MD, Janet BW, Lowe B (2006) A Brief Measure for Assessing Generalized Anxiety Disorder: The GAD-7. Arch Intern Med 166: 1092-1097.
16. Löwe B, Decker O, Müller S, Brahler E, Schellberg D, et al. (2008) Validation and Standardization of the Generalized Anxiety Disorder Screener (GAD-7) in the General Population. Med Care 46: 266-274.

17. Cusin C, Hilton GQ, Nierenberg AA, Fava M (2012) Long-term maintenance with intramuscular ketamine for treatment-resistant bipolar II depression. Am J Psychiatry 169: 868-869.

18. Shiroma PR, Johns B, Kuskowski M, Wels J, Thuras P, et al. (2014) Augmentation of response and remission to serial intravenous subanesthetic ketamine in treatment resistant depression. J Affect Disord 155: 123-129.

19. McIntyre RS, Rodrigues NB, Lee $Y$, Lipsitz O, Subramaniapillai M, et al. (2020) The effectiveness of repeated intravenous ketamine on depressive symptoms, suicidal ideation and functional disability in adults with major depressive disorder and bipolar disorder: Results from the Canadian Rapid Treatment Center of Excellence. J Affect Disord 274: 903-910.

20. Ross C, Rakesh J, Bonnett CJ, Wolfson P (2019) High-dose ketamine infusion for the treatment of post-traumatic stress disorder in combat veterans. Ann Clin Psychiatry 31: 271-279.

21. Krystal JH, Abdallah CG, Sanacora G, Charney DS, Duman RS (2019) Ketamine: a paradigm shift for depression research and treatment. Neuron 101: 774-778.

22. Loo CK, Galvez V, O'Keefe E, Mitchell PB, Pavlovic DH, et al. (2016) Placebo-controlled pilot trial testing dose titration and intravenous, intramuscular and subcutaneous routes for ketamine in depression. Acta Psychiatr Scand 134: 48-56.

23. Mathews DC, Zarate Jr CA (2013) Current status of ketamine and related compounds for depression. J Clin Psychiatry 74: 516-517.

24. Ionescu DF, Fu DJ, Qiu X, Lane R, Lim P, et al. (2020) Esketaminenasal spray for rapid reduction of depressive symptoms in patients with major depressive disorder who have active suicide ideation with intent: results of a phase 3 , double-blind, randomized study (ASPIRE II). Int J Neuropsychopharmacol 24: 22-31. 